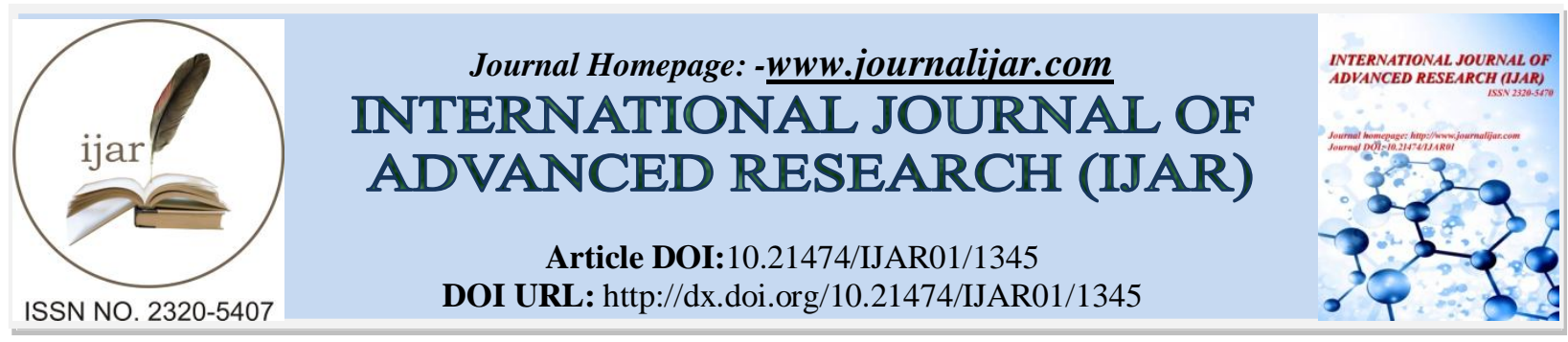

RESEARCH ARTICLE

\title{
DETECTION OF FABRIC DEFECTS.
}

1. M.tech Student, UCoE, Punjabi University, Patiala, India.

Karanveer Singh ${ }^{1}$ and Jaspreet Kaleka ${ }^{2}$

2. Assistant Professor, UCoE, Punjabi University, Patiala, India.

\section{Manuscript Info}

Manuscript History

Received: 18 June 2016

Final Accepted: 15 July 2016

Published: August 2016

Key words:-

\section{Abstract}

Human inspection is the traditional means to promise the quality of fabric. It helps instant correction of small defects, but errors left due to human eye occurs because of fatigue and fine defects are often undetected. Therefore, automated inspection of fabric defect is then a natural way to improve fabric quality and reduce labor costs. The most difficulty faced by industrial inspection problems deals with the textured materials such as textile web, paper, and wood. In order to deal with the problem of defect detection in fabric defect images we have a proposed an efficient algorithm to easily detect the region containing fabric defects. We have converted the input RGB images into other color spaces i.e. Lab and ycbcr which are more close to human perception and gives better results in feature extraction as well as classification stage. For feature detection we have intensity values from these two color spaces in which rough separation of two classes has been evaluated using CSLBP features and DCT algorithms and neural object has been trained and tested to get segment out the defect region from the rest of the fabric image. Experimental results showed better accuracy on various types of defects i.e. hole, broken needle, dye spot, slub defects etc.

Copy Right, IJAR, 2016,. All rights reserved.

\section{Introduction:-}

Fabric is widely used nowadays in every aspect of our life. Textile fibers are usually made of natural element such as cotton or wool; or a compound of different elements such as wool and nylon or polyester. Fabric defect detection is a quality control process that aims at identifying and locating defect of fabric. Human inspection is the traditional means to promise the quality of fabric but errors left due to human eye occurs because of fatigue and fine defects are often undetected. Therefore, automated inspection of fabric defect is then a natural way to improve fabric quality and reduce labor costs. The most difficulty faced by industrial inspection problems deals with the textured materials such as textile web, paper, and wood. The inspection problem present in textured materials becomes texture analysis problems at microscopic levels. Defect detection in web materials normally depends upon identification of regions that differ from a uniform background.

\section{ProposedWork:-}

In this work, we proposed a universal method which uses CS-LBP texture features of the input RGB images after conversion to other color spaces such as Lab and Ycbcr, along with some processing by Discrete cosine transform 
and gives efficiently classify of faulted and standard fabric area in an image. The following are the steps of the procedure:

\section{Step 1:-A 2-D image is loaded in the Matlab Software.}

\section{Preprocessing:-}

- Collection of datasets: All the images were acquired from valid links on World Wide Web and from international journals which are related to fabric defects.

- Pre-processing the fabric defect images: The images were converted to Lab and Ycbcrcolor spaces in which different components i.eL,a,cr components has been used in further processing.

The dimensions in Lab color space are the luminosity $\mathrm{L}$ and the two color components $\mathrm{a}, \mathrm{b}$, coverting red to green and blue to yellow axes respectively. It is a popular model since it not only contains a comprehensive range of colors but also is a linear representation according to human color perception system. Most importantly, errors caused by light can be eliminated when we separate with luminosity and color. Similarly, Ycbcr is also an effective colour space which gives better results than RGB colour space. These colours spaces separate RGB into luminance and chrominance information and hence segment the images with more accuracy

\section{Median filtering:-}

Medfiilt 2 function does median filtering of the matrix A in two dimensions. Each output pixel has the median value in a 3-by-3 neighborhood which is around the corresponding pixel in the input image. On the edges, medfilt 2 pads the image with 0's, so the median values for points within one-half the width of the neighborhood ([rows cols]/2) of the edges which might be shown as distorted. This has been done for local de-noising of the input image which is $\mathrm{L}$ channel of Lab changed image and results in better smooth image than input image.

\section{Step 2:- Gabor filter is applied to it, for edge enhancement.}

Gabor filters, which have been demonstrated to fit well in the receptive fields of the most of the simple cell in the primary visual cortex, are modulation products of Gaussian and complex sinusoidal signals.

- $\lambda$ (Wavelength=8):This is the wavelength of the cosine factor of the Gabor filter kernel and here with the favorable wavelength of this filter.

- $\Theta($ Orientation $=0-180)$ : This parameter specifies the orientation of the standard to the parallel stripes of a Gabor function.

- $\Phi$ (Phase offset $=0$ ): The phase offset $\varphi$ in the argument of the cosine factor of the Gabor function is specified in degrees. Valid values are real numbers between -180 and 180.

- $\gamma$ (Aspect ratio): This parameter, called more exactly the spatial aspect ratio, specifies the ellipticity of the carry of the Gabor function.

- $\mathrm{b}$ (Bandwidth=5): The half-response spatial frequency bandwidth $\mathrm{b}$ (in octaves) of a Gabor filter is in relation to the ratio $\sigma / \lambda$, where $\sigma$ and $\lambda$ are the standard deviation of the Gaussian factor of the Gabor function and the favourable wavelength.

Step 3:- Further by Discrete Cosine Transform (DCT) function frequencies are segmented. A block-based transform and which can be conducted parallelly is called Discrete Cosine Transform (DCT). We investigate the coding performance of 2d- DCT transform based image coding approach, where the transformed coefficients are filtered by two means. In first, we keep the low value coefficients and in second we keep high value coefficients. Then inverse DCT has been applied to get the images in time domain. After this, these two images are multiplied for further processing.

Step 4:- A function Centre-Symmetric Local Binary pattern (CSLBP) is put to use, to find the center value of the whole image, which is further assigned to a variable. This function takes patch or image as input and return Histogram of CSLBP operator.

It is a enhanced form of local binary pattern. It separates a local pattern for every pixel of the input image region. Same as LBP, in CSLBP, each pixel is regarded as center pixel. Distinction of center symmetric pixels is measured which is not dependent on the center pixel. Based on the difference, four binary numbers are allotted to the center pixel that is then multiplied by four weights, and collectively gives one value, that is called center symmetric local binary pattern value. CSLBP is explained mathematically in the following equations. 


$$
\begin{aligned}
& C S L B P_{P, R, T}=\sum_{s=0}^{(p / 2)-1} 2^{5} \times T_{3}\left(I_{s}-I_{s+(p / 2)} \ldots \ldots \ldots \ldots . .(1)\right. \\
& T_{3}= \begin{cases}1 & a>T \\
0 & \text { else }\end{cases} \\
& \left.H(L)\right|_{C S L B P}=\sum_{x 1=1}^{m} T_{2}\left(C S L B P\left(x_{1}, x_{2}\right), L\right) ; L \varepsilon[0,15]
\end{aligned}
$$

Where $\mathrm{T}$ is the threshold parameter and its value is set to $1 \%$ of the highest intensity in the image. An example of CSLBP is explained in Figure below

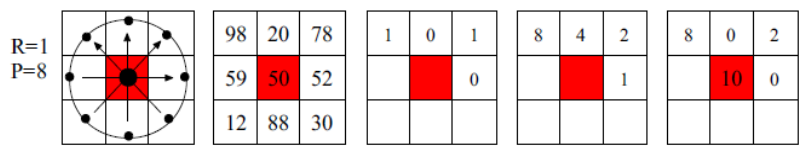

Fig1:- Center symmetric local binary pattern computation example [2]

Step 5:- A block_struct() is used to work on block pixels according to the value of block given like(24,24) $\mathrm{B}=$ blockproc(A,blockSize,fun) evaluates the image $\mathrm{A}$ by using the function fun to each different block of $\mathrm{A}$ and joining the obtained results into B, the output matrix. blockSize is a two-element vector, [rows cols], that states the size of the block.fun is a handle to a function that receive a block struct as input and returns a matrix, vector, or scalar Y. For example, Y = fun (block_struct). For each block of data in the input image, A, blockproc passes the block in a block_struct to the user function, fun, to give $\mathrm{Y}$, the corresponding block in the output image. If $\mathrm{Y}$ is empty, blockproc does not produce any output and gibes back an empty after processing all blocks. Choosing an appropriate block size can significantly improve performance. In this we make fun function based on entropy of the blocks and $24 * 24$ size has been chosen for the blocks.

\section{Step 6:-fcm function is applied to entropy of the image after CSLBP, and divides it into three clusters.}

The fuzzy C-Means (FCM) [3] algorithm is an unsupervised classification scheme, and has been widely studied and applied in some various evident areas. Because fast FCM algorithm is appropriate to fall into the local optimization and where fast FCM algorithm can find best is hugely dependent on the initialization. We have used this to get the segmentation of entropy based image into three clusters in which that cluster has been used which contains minimum number of pixels. So image binarization has been carried out further to keep that cluster in one class and rest in other class. After this erosion and dilation operations has been carried out to get the largest binary object. This is explained in next step.

\section{Step 7:- In this step, erosion is done to abrade the boundaries of foreground pixels.}

Morphological Erosion is one of the fundamental operators in the field of mathematical morphology. The basic effect of the operator on a binary image is to abrade away the boundaries of regions of foreground pixels. Thus areas of foreground pixels reduce in size, and holes within those areas become bigger. Erosion has been used to get rid of the unwanted pixels present next to the defected region and highlight the defected pixels. Thresholding followed by skeletonizing and thinning has not been used as it removes the important pixels and the output retrieved isn't as efficient. A structuring element used in this work is

se1 $=\operatorname{strel}(\operatorname{eye}(5))$;

im1=imerode(im,se1);

The value of the output pixel is the minimum value of all the pixels in the initial pixel's neighborhood. In a binary image, if any of the pixels is set to 0 , the output pixel is set to 0 . Pixels beyond the image border are assigned the maximum value afforded by the data type. For binary images, these pixels are supposed to be set to 1 . For gray-scale images, the maximum value for image is 255. Similarly, dilation is just opposite to erosion algorithm.

\section{Step 8:- Training and testing of the two classes by ANN.}

Most done work regard the Back Propagation network to be the quintessential Neural Net. Actually, Back Propagation is the training or learning algorithm than the network itself. The procedure of determination of the error rates of each neuron that affect the output is called back propagation. The neurons of the input layer are fully attached to the hidden layer and the results are fully joined to the output layer. A Back Propagation network can be learned by an example. One can give the algorithm examples of what you want the network to do and it gives different network's weights, so that, when training is completed, it will give you the needed output for a particular 
input. The input and its according with target are called a Training Pair. Back Propagation networks are useful for simple Mapping and Pattern Recognition Tasks. As just stated, to train the network you require to give it examples of what is required - the end result you want (called the Target) for a particular input.

In this work, we have differentiated the training data into two rough classes in which majority of faulty portion comes in one class and majority of the correct fabric pixels in another class. As the purpose behind using neural network is that it gives better accuracy when tested on the similar or same image as it trained the neurons well even when two distinguished classes are not that accurate. Therefore, after testing we get the better segmented image than the input image.

\section{Resultsand Discussions:-}

For assessing the performance of the suggested algorithm we have collected a wide variety of fabric defects in which dye spot, holes, ladder, slub, slack warp, slack end, oil mark etc. has been considered. Few of the images from the collected dataset has been shown below

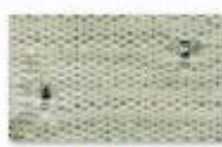

Holes

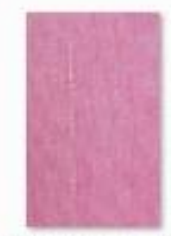

weaving-defect

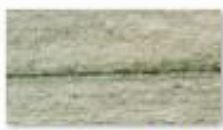

Missing Plush

Loop

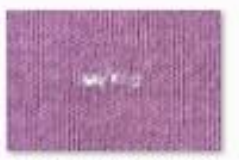

Fabrics-Defects1

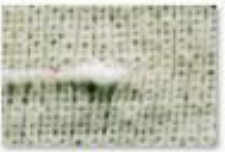

Slub

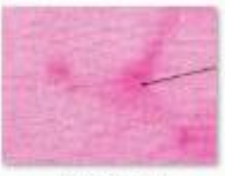

Dye Spot

Fig 1.2.1:- A screen shot from the collected dataset.

The results in terms of output images at different steps has been described below

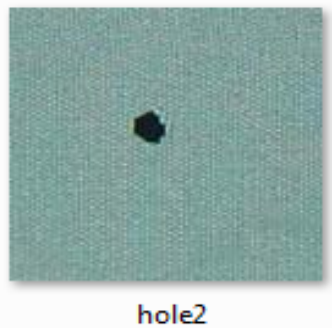

Fig 1.2.2:- Input fabric image containing hole as a defect.

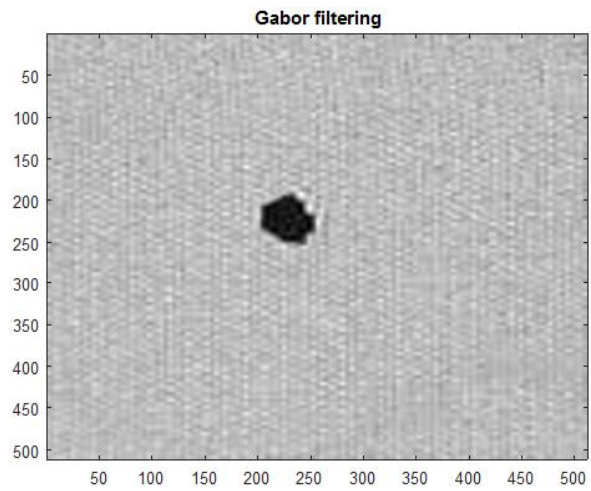

Fig 1.2.3:- $\mathrm{L}$ channel of Lab converted image after Gabor filtering. 


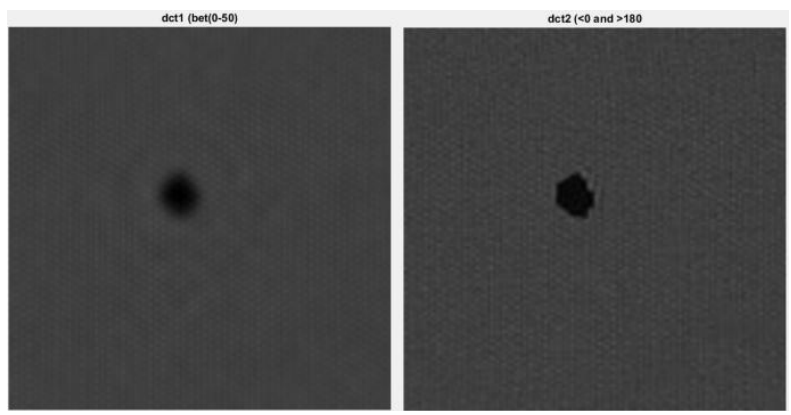

Fig 1.2.4:- (a) DCT transform image containing coefficients between 0 and 50 (b) DCT transform image containing coefficients below 0 and above 180

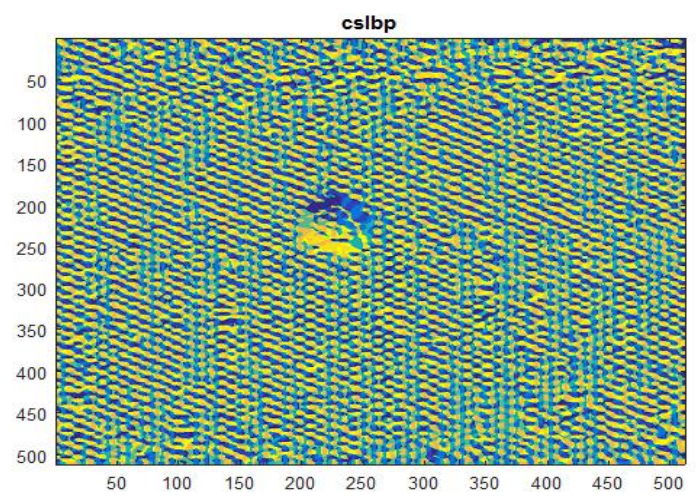

Fig 1.2.5:- Image after CSLBP evaluation

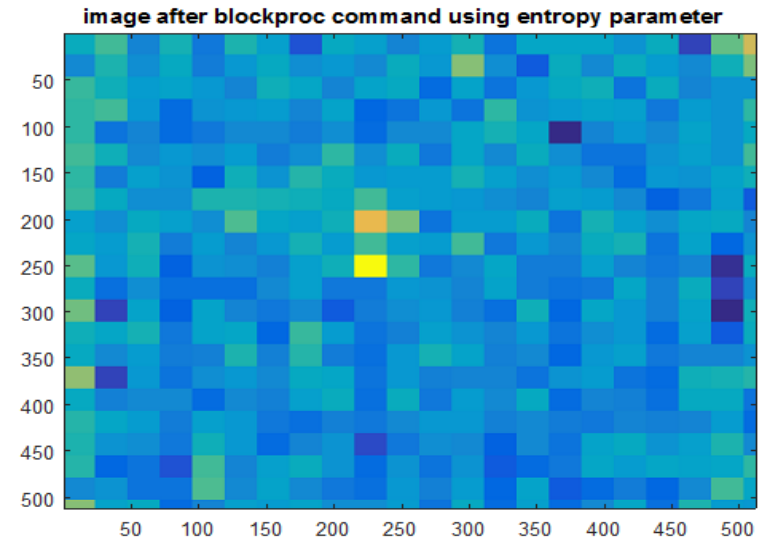

Fig 1.2.6:- Image after getting entropy of $24 * 24$ image blocks

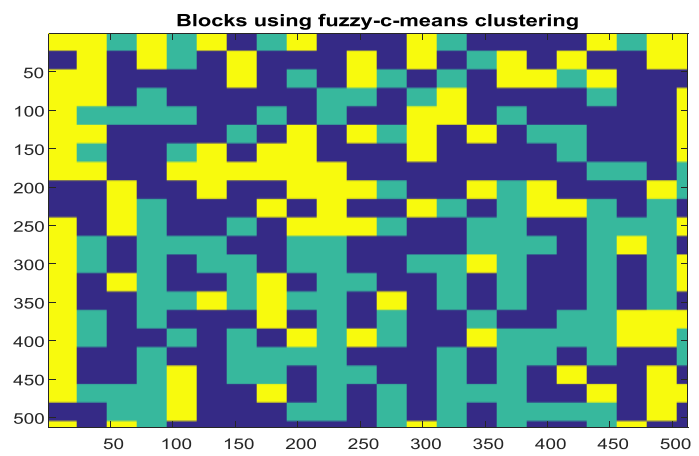

Fig 1.2.7:- Image after FCM clustering 


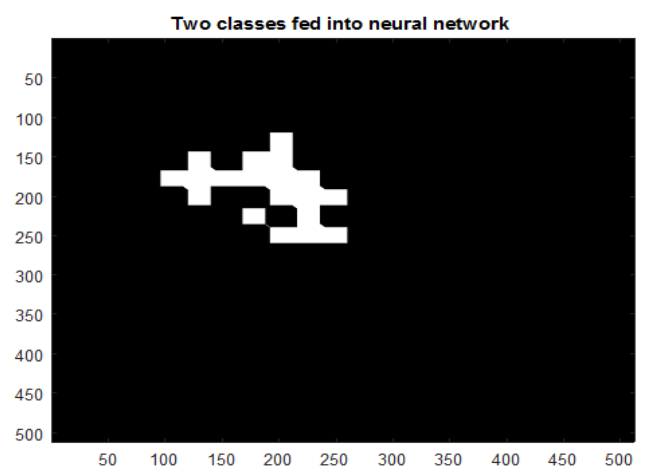

Fig 1.2.8:- Image after making rough classes for neural network input.

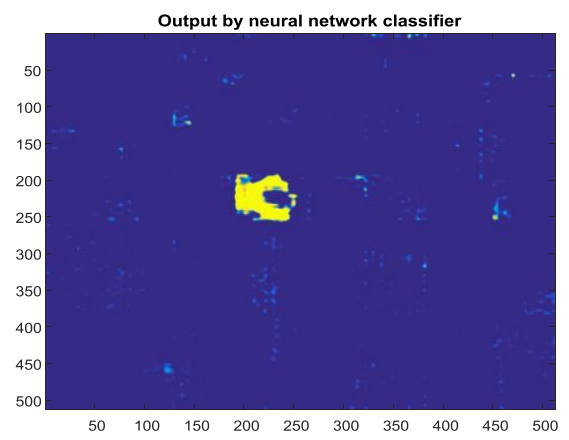

Fig 1.2.9:- Output after testing the image by neural network object

From above image it has been found that defect has been separated from the rest of the fabric image and has been shown in high intensity pixels. Similarly results for other images has been shown below

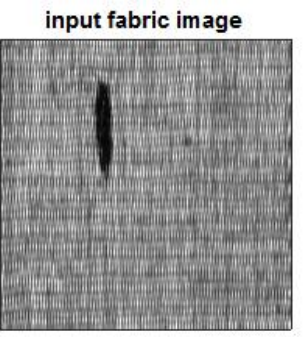
Output by neural network classifier

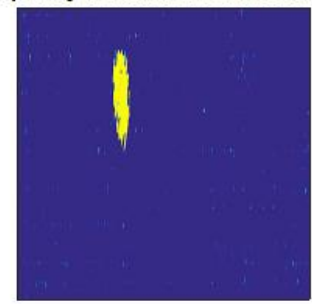

Fig 1.2.10:- (a) Hole 2 defect image; (b) hole defect segmented image

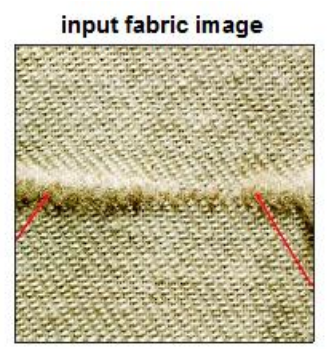

Output by neural network classifier

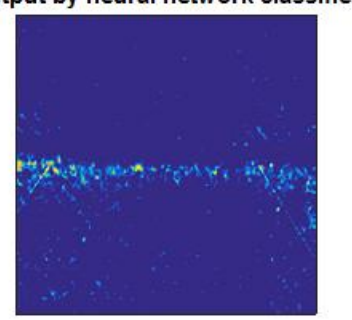

Fig 1.2.11:- (a) Slough-off Weft defect image (b) Slough-off Weft defect segmented image 

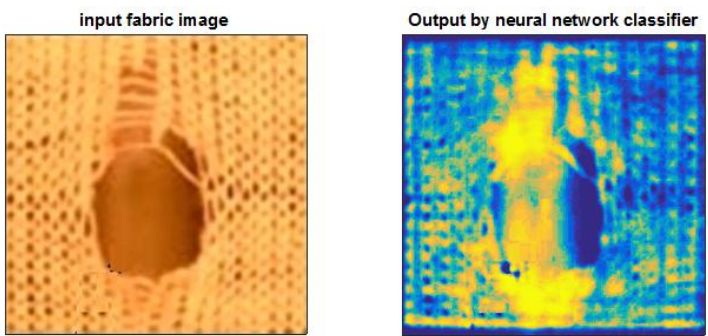

Fig 1.2.12:- (a) Hole and broken needle defect image (b) Hole and broken needle defect segmented image.
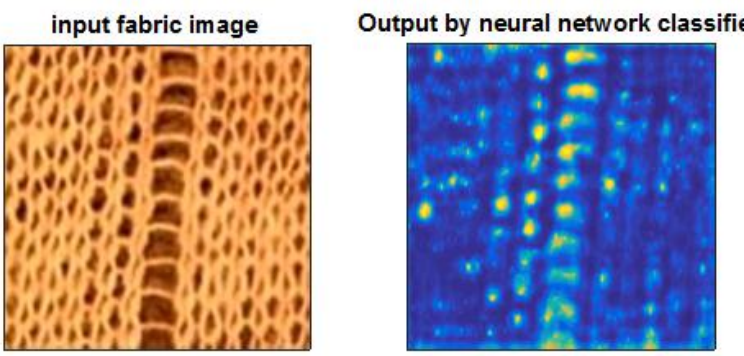

Fig 1.2.13:- (a) broken needle defect image (b) broken needle defect segmented image.
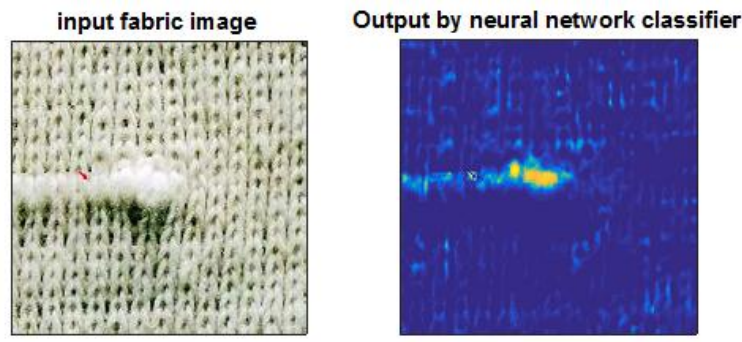

Fig 1.2.14:- (a) slub defect image (b) slub defect segmented image.
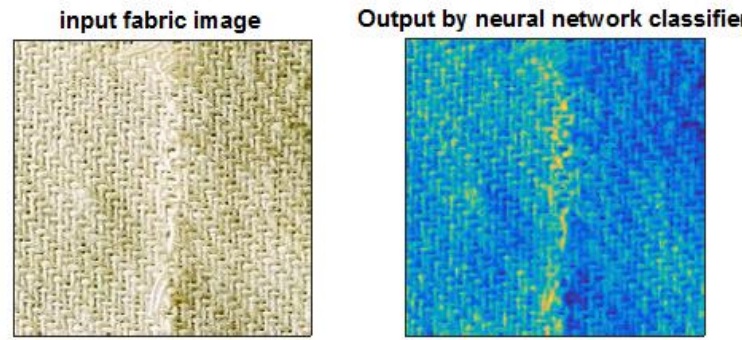

Fig 1.2.15:- (a) slack end defect image (b) slack end defect segmented image.
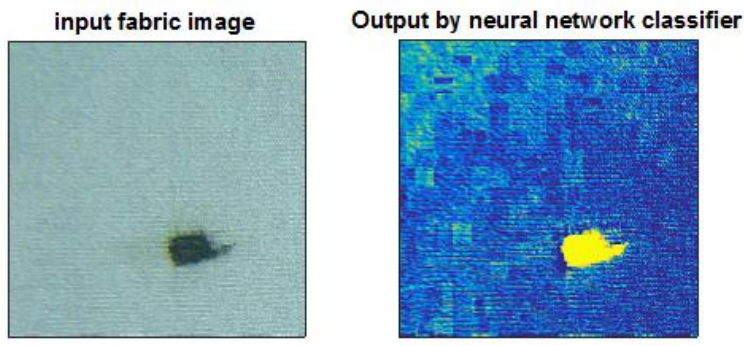

Fig 1.2.16:- (a) oil stain defect image (b) oil stain defect segmented image 


\section{Conclusion:-}

In present work, we have proposed a neural network based segmentation system which classifies the pixels of the defect image into normal region and defected region. The usage of lab and ycbcr color spaces and CSLBP features have been extracted from the L channel of lab converted image. DCT transform has been applied in order to filter out high and low frequencies in the image and further their output has been considered and CSLBP features have been taken from them. Further block based entropy values has been generated on which clustering has been applied which segments the image into limited number of clusters, Further, connected component analysis has been carried to make two rough classes in which one class contains majority of defect pixels in it. In the end neural classification has been carried out for final segmentation of defect region in the input image.

\section{References:-}

1. ManishaVerma et al., (2015) "Center symmetric local binary co-occurrence pattern for texture, face and biomedical image retrieval" Published in J. Vis. Commun. Image R. 32 224-236

2. Bedzek J.C. (1981) "Pattern Recognition with Fuzzy Objective Algorithm" New York.

3. S.N. Sivanandam et al., "Introduction to Neural Networks using MATLAB 6.0", TMH, 20(116). 